\title{
Palingenesi e sogno nel primo Onofri
}

\section{Massimo Maggiari}

L'esordio poetico di Arturo Onofri giunge nel marzo del 1907 con la prima ed unica edizione di Liriche. La raccolta di poesie preliminarmente porta la seguente premessa, datata Roma, 16 febbraio 1907:

O mio libro, ti scaglio lontano da me.

Più non ti voglio, né più ti conosco per mia creatura. Troppo sei antico perché tu possa cantare ancora dell'anima mia che si muta ogni giorno (oh quanto ora diversa da quella che cantò la più antica di queste liriche!).

Libro io ti distruggo e ti getto. M'è necessario sacrificarti sull'ara della mia Volontà perché tu ceda l'aria e la luce ad altre più belle creature che già m'ho composte.

Non imito, io, forse, in questo sacrificio l'opera sempre nova dell'eterna Natura? (5)

In questo rifiuto fittizio della paternità letteraria della prima opera onofriana risaltano alcuni elementi poetici fondamentali: la poesia intesa come canto, il ruolo centrale dell'anima e la coscienza della durata delle cose, ovvero del loro essere nel tempo e della loro trasformazione. Questo ultimo aspetto è messo in rilievo dal poeta-critico Roberto Mussapi che sottolinea in Onofri il tentativo di cogliere un rapporto essenziale tra vita e tempo sviscerando il nucleo pulsante dell'essere nella sua stessa durata (Il centro e l'orizzonte 31-46).

Altro elemento importante ed esterno di questa raccolta è il frontespizio. A conferma del clima crepuscolare che pervade la prima poesia onofriana, al centro della copertina si staglia un sole calante, figura emblematica sul quale il poeta insiste inserendo un puntino centrale alle " $\mathrm{O}$ " del proprio nome e cognome in modo da rapportarle al simbolo del sole.

Dopo questa prima sottolineatura solare nel frontespizio, in Liriche Onofri ci propone l'immagine del sole morente nell'ottava poesia, intitolata "A. M. Gorki". Divisa in due parti, ognuna consistente in un sonetto, questa composizione rappresenta un omaggio allo scrittore russo allora in esilio all'isola di Capri (19071913) dopo la fallita rivoluzione russa del 1905. Il poema è pervaso da un diffuso titanismo di chiaro stampo nietzschiano, "ei nato in un deserto da leone" (1.11), da cui tuttavia si svincola con sorpresa l'ultima strofa che, sfuggendo la retorica dell'encomio, prelude all'Onofri migliore:

Ed il fanciullo affiso al sol morente col trepido occhio gonfio di mistero, pensa già il sole delle nuove aurore. $(2.12-14)$ 
Nel ventaglio dei riferimenti letterari, che vanno dal crepuscolare "sol morente" alla pascoliana figura del fanciullo, rivendica una posizione centrale la contemplazione del mistero. Secondo Laura Lepri, nel primo Onofri il mistero rappresenta il richiamo ad un'elevazione spirituale che, tramite il dettato poetico, tende a farsi messaggio catartico e palingenetico. Infatti il crepuscolarismo di Onofri non si esaurisce in se stesso, in una poetica di una condizione di crisi e di una vitalità in estinzione, ma al contrario apre il fanciullo ad un enigmatico noumeno, di cui la notte risulta il simbolico suggello:

le Verità che nella densa notte

le pupille, disperse nella via

immensa, invan tentarono ... Ah follia!

(“La canzone dei passeri”" 20-22)

È questa, però, un'apertura che si dimostra velleitaria. Nonostante la dimensione notturna risulti alle "pupille" "densa" e pregna di verità, segnalando forse una partecipazione o una coappartenenza iniziale del noto all'ignoto, all'io lirico non resta che l'amara consapevolezza dell'incapacità di tradurre in esperienza l'esigenza conoscitiva. ${ }^{1}$

Questo senso d'inadeguatezza non rappacifica certo la fondamentale tensione gnoseologica della nascente poesia di Onofri che converge, dopo aver individuato come ostacolo la stessa condizione terrena, nel richiamo ad un progetto di elevazione spirituale per l'uomo:

\author{
... Anima mia, \\ scuoti la polve delle passioni, \\ che troppo è grave alle tue ali il pondo; \\ sperdila al vento o gettala in balia \\ del volgo turpe in tutte abiezioni: \\ tu libera volar devi sul mondo! ("Esortazione" 9-14)
}

Spetta all'anima il compito di sollecitare le energie adeguate a promuovere il proprio riscatto, e quindi la propria spiritualizzazione, propiziando nell'immagine del volo eterico non tanto la conquista fisica delle forze della gravità quanto soprattutto una conquista simbolica dell'anima stessa.

In tale modo il bisogno di elevazione si attua nel trapasso dell'anima dalla terra al cielo, realizzando così una totale separazione dall'elemento terreno e quindi una palingenesi dell'anima. Considerando però che tale bisogno di elevazione origina da un'esigenza conoscitiva, è importante, a mio parere, indagare il significato simbolico-gnoseologico dell' immagine del volo. Il librarsi dell'anima sul mondo rimanda, infatti, al punto di vista diverso, se non oggettivo, di chi contempla il tutto dall'ottica delle stelle e del cielo. ${ }^{2}$ Con l'assunzione di quest'ottica la visione dell'anima liberata corrisponde a una visione totale ed immediata del mondo che si proietta al di là delle limitazioni della visione soggettiva e 
terrena, vagheggiando un'oggettività che rimanda in modo speculare a se stessi. E contemplare se stessi e il mondo non rappresenta forse il tentativo di penetrare quella parte di sé in ombra nel tentativo di assegnare alla specularità, assunta a processo conoscitivo, una funzione purificatrice? ${ }^{3}$

In Liriche il bisogno di elevazione spirituale del poeta si traduce nondimeno, per metonimia, in una serie di immagini in cui si attua la sublimazione dell'elemento terreno tramite un processo fisico di esalazione o di evaporazione nell'etere:

Come quando da occulto avvampamento

s'eleva, primo, un esile opalino

fumo che va nel limpido mattino

verso l'immenso azzurro firmamento

così dall' antro, dove il mio destino

medita d'ogni semplice elemento

della Terra feconda un suo portento

volubile salìa nel cielo latino

già un fume tenue dalla prima brace. ("Dall'antro" 5-9)

Tramite questa volatilizzazione ed elevazione in immagini poetiche dell'elemento materiale, secondo lo studioso junghiano Edward Edinger, si compie nell'immaginario un'operazione di sublimatio alchemica di grande valore psicologico. Il movimento di ascensione favorisce infatti una strategia di distanziamento che, elevando il poeta ad un punto di vista superiore, ne facilita una visione più ampia e quindi emotivamente meno coinvolgente di problemi concreti e personali. Se esaminiamo psicologicamente i versi onofriani della composizione "Dall'antro", il fumo che "va nel limpido mattino" può raffigurare un bisogno di proiettarsi nell' "immenso azzurro firmamento" probabilmente per fugare un sentimento depressivo di chiusura e limitazione, come suggerisce l'immagine dell'antro/tana. In questo modo il bisogno di elevazione rivela soprattutto un bisogno di catarsi psicologica che il poeta sembra ricercare tramite la mediazione fantastica dell'atto poetico:

Canzone, come un tenue fil di fumo

dal mio cuore all' azzurro, ecco, tu sali lieta se ti disperda un frullo d'ali.

("La canzone dei passeri" 91-93)

Fondamentalmente l'impeto poetico, per mezzo della proiezione fantastica, aiuta l'io lirico a staccarsi da quelle "sottili radici velenose "che impediscono all'anima di spiccare il volo generando angoscia e conflitto:

Non potrò sgrovigliare

dunque, l'arteriale

intrico di sottili 
radici velenose

in che rinchiusi al volo

l'ale desiderose

come in tenace rete? ("Inanis flagrans" 3.11-17)

In Memories, Dreams, Reflections Carl Gustav Jung sostiene che distacco ed oggettività sono le caratteristiche essenziali del sogno ed il segreto fondamentale del processo di individuazione. ${ }^{5}$ Jung afferma anche che la cognizione oggettiva della psiche, manifestata nella rappresentazione del sogno, è necessaria in quanto aiuta a differenziarsi dalle proiezioni psicologiche che legano emotivamente l'individuo al mondo. Questo significa che tali proiezioni rendono soggettiva, in un senso negativo, la visione della realtà favorendo una percezione riduttiva e distorta del mondo.

Nel giovane Onofri la serenità dell'io poetico è spesso insidiata in modo particolare dalle passioni della carne, a tal punto che in Liriche, nei versi di "Orrore", il poeta confessa la propria disperazione dopo un'esperienza di amore mercenario:

or che sei sazia nella mattutina

ebrietà di sonno, si fa nota

al mio disgusto che dal cuor sornuota

ogni tua frode immonda, o concubina.

Le impurità de' tuoi venduti amori

passano in rombo sulle turbe ladre

e te io odio, simbolo giacente. ("Orrore" 5-11)

In tale clima poetico ed umano il distacco conseguito col volo favorisce l'evasione da un sentimento di sopraffazione che grava sull'io poetico e che, per generalizzazione, il poeta estende alla condizione terrena. Il motivo del volo soddisfa pertanto un bisogno di allontanamento da una conflittualità che probabilmente deriva dall'incapacità del poeta di controllare le pulsioni sessuali. Di conseguenza Onofri non è tanto interessato a conciliare materia e spirito, piano terreno e piano cosmico, quanto a fuggire dalla materialità, e quindi dal sentimento d'angoscia generato dagli istinti, rifugiandosi nella spiritualità della dimensione eterea. $^{6}$

La poesia e le sue figure mediatrici (la madre pia e consolatrice, il cavallo alato, l'anima che vola) contribuiscono pertanto a un processo fantastico di eterealizzazione che promuove una condizione di idillica serenità a cui l'io lirico aspira, al punto di fondere il motivo del volo a quello del sogno:

Al limite del bosco

Anima mia, reclina

dormi sulla novella

erbetta smeraldina,

respirando gli effluvi 


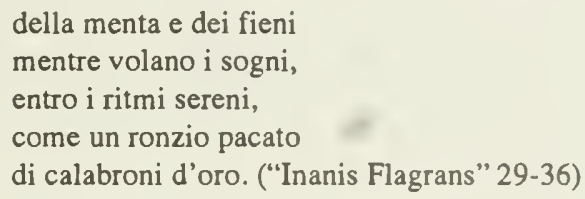

In questa prospettiva, il successivo rapporto stabilito dal poeta tra l'io lirico, la donna e la poesia non fomenta più alcun conflitto, ma al contrario addita un preciso connubio simbolico che, al di là dell'eliminazione degli opposti, conferisce un carattere spirituale e femminile alla poesia stessa. Con la visione della donna "raccolta nelle spire del canto", Onofri contrappone infatti alla femminilità adulterata e deprimente della prostituta l'immagine verginale e raccolta della donnapoesia esaltando un clima stilnovistico di angelica e schiva purezza:

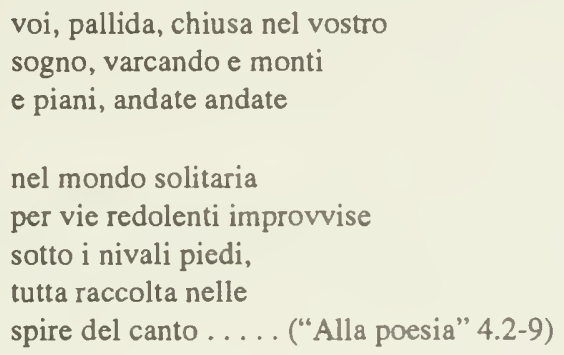

L'estrema introversione che caratterizza l'immagine della donna ("voi, pallida, chiusa nel vostro sogno") effettua un distacco dalla realtà, concentrando quindi quella figura femminile sul proprio mondo immaginario e salvaguardandola da eventuali dispersioni esterne. Questa tendenza al contenimento psicologico incoraggia un ritorno alla radice immaginale del mondo svelando, come del resto fa il sogno, in quanto attività onirica, una fonte d'intelligenza che congiunge ciò che è essenziale all'immaginazione. Entro questa prospettiva, la visione della figura che va "varcando e monti e piani" ci riporta alla proiezione speculare di chi pone di fronte a sé il mondo come propria oggettività nel tentativo di penetrare la realtà nella sua essenza noumenica. Da questa tensione conoscitiva emerge la poesia del giovane Onofri; poesia che suscita la contemplazione di una profonda ed essenziale entità che, congiungendo ciò che è fuori a ciò che è dentro, restituisce l'individuo alla propria anima ed al mondo.

\section{College of Charleston}

\section{NOTE}

1 Per quanto riguarda il concetto di mistero in relazione alla problematica del conoscere e della conoscenza simbolica si veda Sini 78-83.

2 Sullo sguardo che parte dai cieli in relazione all'impresa poetica dell'io si veda Mussapi, 
"Shakespeare".

3 Immedesimandosi al movimento del volo l'anima si riappropria della sua natura eterea incarnando le qualità più congeniali alla contemplazione del mondo: grazia e mobilità. Un'esauriente analisi dell'immagine del volo che ne mette in evidenza la generale fenomenologia si trova in Bachelard 19-89. Per quanto riguarda invece il processo di autoriconoscimento si veda D'Alessio.

4 Per un approfondimento dei significati psicologici e simbolici delle operazioni alchemiche, tenendo in particolare considerazione l'operazione di sublimatio, si vedano i seguenti studi: Edinger, Franz, Alchemy; Zolla, Le meraviglie.

5 Sul concetto di cognizione oggettiva specialmente in relazione ai sogni che precedono la morte si vedano: Franz, On Dreams 114-15; Jung, Erinnerungen Träume Gedanken nella traduzione in inglese a cura di Aniela Jaffe: Memories, Dreams, Reflections 295-98. Sul concetto di cognizione oggettiva in relazione al processo d'individuazione si veda invece Carotenuto 86-99.

6 Per una maggiore comprensione del significato spirituale della fenomenologia aerea si esaminino le osservazioni di Jung sul termine "spirito". Per "spirito", afferma lo studioso, si intende in generale una sostanza immateriale o una forma di esistenza che si colloca in opposizione alla materia e che nelle sue forme e livelli più universali è chiamata Dio. Jung continua osservando che la natura dello spirito è simile a quella del vento, in quanto lo spirito è sempre attivo, alato e veloce, in movimento, e in tutto ciò che vivifica, stimola, incita ed ispira (Jung, The Archetypes 34-42).

7 Nella donna-poesia è riconoscibile una figurazione di Artemide/Diana. Non è casuale tale figurazione se consideriamo che Artemide è la sorella di Apollo e l'archetipo di una femminilità che è assoluta, pura e primitiva. Queste considerazioni suggeriscono che un certo tipo di poesia affiora da una zona essenziale, e quindi vergine, della psiche umana. Per un approfondimento di tale archetipo si vedano: Paris 109-63; Hillman 129-45; Zolla, L'amante 105-17.

\section{OPERE CITATE}

Bachelard, Gaston. Air and Dreams. Dallas: Dallas Institute, 1988.

Carotenuto, Aldo. Il labirinto verticale. Roma: Astrolabio, 1981.

D'Alessio, Carlo. "Le metamorfosi di Narciso. Simbologie e strutture dell'autoriconoscimento nella poesia di Arturo Onofri". Galleria 39 (1989): 352-69.

Edinger, Edward. Anatomy of the Psyche. Alchemical Symbolism in Psychotherapy. La Salle: Open Court, 1985.

Franz, Marie-Louise von. Alchemy. An Introduction to the Symbolism and the Psychology. Toronto: Inner City Books, 1980.

. On Dreams and Death: A Jungian Interpretation. Trans. Emmanuel Xilopi. Boston: Shambala, 1987.

Hillman, James. Anima. An Anatomy of a Personified Notion. Dallas: Spring, 1985.

Jung, Carl Gustav. The Archetypes and the Collective Unconscious. Princeton: Princeton UP, 1981.

. Memories, Dreams, Reflections. Ed. Aniela Jaffe. New York: Vintage, 1973.

Lepri, Laura. "Onofri edito ed inedito". Studi novecenteschi 27 (1984): 55-70.

Onofri, Arturo. Liriche. Roma: Vita letteraria, 1907.

Mussapi, Roberto. "Shakespeare e la cancellazione dell'io". Tradurre poesia. Brescia: Paideia, 1983. 252-58.

Il centro e l'orizzonte. La poesia in Campana, Onofri, Luzi, Caproni, Bigongiari. Milano: Jaca Book, 1985.

Paris, Ginette. Pagan Meditations. Aphrodite, Hestia, Artemis. Dallas: Spring, 1986.

Sini, Carlo. Il profondo e l'espressione. Filosofia, psichiatria, e psicanalisi. Milano: Lanfranchi, 1991. 
Zolla, Elemire. L'amante invisibile. L'erotica sciamanica nelle religioni, nella letteratura e nella legittimazione politica. Venezia: Marsilio, 1986.

. Le meraviglie della natura. Introduzione all' alchimia. Venezia: Marsilio, 1991. 